\title{
Aplicaciones Móviles para Mejorar la Comunicación entre Docente y Alumno
}

\author{
Marín Lozano, Erasto Alfonso \\ Universidad Veracruzana, México \\ emarin@uv.mx
}

\author{
López Lunagómez, Daniel Antonio \\ Universidad Veracruzana, México \\ dlopez@uv.mx
}

Resumen - Actualmente vivimos en la era de contantes avances tecnológicos la cual ha llevado a la contante creación de dispositivos que evolucionan contantemente introduciendo los nuevos avances. Debido a ello las mayorías de las personas cuentan con un dispositivo para su uso diario creando una gran dependencia y haciéndolos indispensables para la vida diaria. Los Smartphones o teléfonos inteligentes, es un dispositivo móvil que ofrece capacidades computacionales y de conectividad más avanzadas que los teléfonos móviles convencionales. El desarrollo de una aplicación que solucione algunos de los problemas que se presentan en la misma, centrado esta vez en los problemas de comunicación entre los estudiantes y maestros en los planteles.

Palabras Clave: Smartphones; Aplicación móvil; Apps; Sistemas operativos; Bases de Datos para aplicaciones móviles; Programación en Android;

\begin{abstract}
Today we live in the era of fierce technological advances which has led to the counting creation of devices that are evolving considerably introducing the new advances. Because of this, most people have a device for daily use creating a great dependence and making them indispensable for daily life. Smartphones are a mobile device that offers more advanced computing and connectivity capabilities than conventional mobile phones. The development of an application that solves some of the problems that arise in it, this time focused on communication problems between students and teachers in the campuses.
\end{abstract}

Keywords: Smartphones; Mobile app; Apps; Operating systems; Databases for mobile applications; Android programming;
Interconectando Saberes, 2019

ISSN: 2448-8704
Fecha de Recepción: 14 de septiembre de 2019

Fecha de Aceptación: 18 de octubre de 2019

Fecha de Publicación: 31 de octubre de 2019 


\section{INTRODUCCIÓN}

Actualmente los Smartphones se han convertido en algo indispensable para a vida diaria, con dispositivos que usamos todos los días y se han convertido en una necesidad, pero ello es indispensable el conocer más a fondo acerca de estos dispositivos, así como de las aplicaciones que nos brindan.

Una de las características más importante de los teléfonos inteligentes es que permiten instalar diversos programas que permiten incrementar el número de operaciones que se pueden realizar, programas que actualmente son mejor conocidos como aplicaciones o apps.

Estas mencionadas apps pueden ser desarrolladas por el fabricante del dispositivo las cuales son comúnmente conocidas como aplicaciones predefinidas o nativas del dispositivo, también pueden ser desarrolladas por el operador o un tercero las cuales pueden ser descargables desde las tiendas de aplicaciones de cualquier dispositivo.
Las aplicaciones predefinidas o nativas de un dispositivo son aplicaciones que se encuentran siempre en todos los teléfonos inteligentes desde su compra como lo son las siguientes: Llamadas, mensajes, contactos, multimedia, cámara, reloj, correo, visualizador de documentos, calendario, navegador, tienda.

El artículo se realizó a través de una revisión de fuentes bibliográficas tanto impresas como electrónicas sobre toda la información que se consideró necesaria para comprender el tema de estudio, que se desea profundizar.

\section{MARCo TEÓRICO \\ Smartphone}

Lozano Mauricio (2018) Un smartphone es en su traducción literal del inglés un "Teléfono Inteligente" Lo que hace inteligente a un teléfono es la capacidad de llevar a cabo otras funciones además de realizar llamadas telefónicas y enviar mensajes. Muchas de estas características tales como enviar y recibir correos o editar documentos de office, antes sólo las habrías llevado a cabo utilizando una computadora. 
En términos generales un smartphone es un celular con un robusto sistema operativo, aplicaciones y conexión a internet. Pero para entender por completo todas las características que hacen inteligente a un smartphone está esta guía (Lozano, 2018).

En un inicio existían los teléfonos celulares y como un dispositivo adicional los asistentes digitales personales, también conocidos como "Palm".

Los teléfonos celulares se utilizaban sólo para realizar llamadas y las Palm podían almacenar datos de contacto y listas de tareas pendientes - y sincronizarse con la computadora. Eventualmente las Palm obtuvieron conectividad inalámbrica y llegaron a enviar y recibir correos electrónicos. Los teléfonos celulares por su parte metieron mano en el campo de mensajería y posteriormente empezaron a agregar más características de los asistentes personales hasta convertirse en Smartphones (Lozano, 2018).
Características clave en un Smartphone

Aunque no hay una definición estándar del término "Smartphone" en toda la industria, creímos relevante señalar lo que las tendencias indican que es un Smartphone (Lozano, 2018).

\section{Sistema Operativo}

Todos los smartphones son ejecutados a través de un sistema operativo. Este mismo permite correr distintos tipos de aplicaciones. Los teléfonos iPhone operan a través de un sistema operativo llamado iOS, los BlackBerry a través de BlackBerry OS, los HP a través de WebOS, los Windows Phone a través de un sistema de Microsoft para móviles y el más popular y utilizado por decenas de marcas, Android de Google (Lozano, 2018).

\section{Apps}

Aunque casi todos los celulares convencionales ya incluyen algún tipo de software (incluso los modelos más básicos, con libreta de direcciones o gestor de contactos), un teléfono inteligente tiene la capacidad de hacer mucho más. Las aplicaciones son pequeños progra- 
mas creados específicamente para dispositivos móviles y permiten obtener una experiencia más adaptada y más fluida cuando se accede a través de un smartphone. Por ejemplo, Facebook, fácilmente podríamos acceder desde nuestro smartphone utilizando el explorador, pero la experiencia no es para nada parecida a la que obtenemos al utilizar la aplicación.

\section{Navegación Web}

Utilizar tu smartphone para navegar en internet no es algo que siempre haya estado disponible, es otra de las grandiosas características que ofrece un smartphone. Claro, basado en las características de tu dispositivo puedes obtener velocidades más altas, pero en general todos los smartphones de hoy en día permiten navegar cuando menos en redes $3 \mathrm{~g}$. Las aplicaciones son altamente útiles, pero no siempre son necesarias, para todo este tipo de situaciones existe el explorador web (Chrome, Opera, Safari, etc.).
Aplicaciones (APPS)

Cuello Javier y Vittone José (2017) nos dicen que, aunque no parezca, las aplicaciones llevan tiempo entre nosotros. Antes de empezar a diseñarlas, Las aplicaciones (también llamadas apps) están presentes en los teléfonos desde hace tiempo; de hecho, ya estaban incluidas en los sistemas operativos de Nokia o Blackberry años atrás. Los móviles de esa época contaban con pantallas reducidas y muchas veces no táctiles, y son los que ahora llamamos feature phones, en contraposición a los smartphones, más actuales.

En esencia, una aplicación no deja de ser un software. Para entender un poco mejor el concepto, podemos decir que las aplicaciones son para los móviles lo que los programas son para los ordenadores de escritorio. Actualmente encontramos aplicaciones de todo tipo, forma y color, pero en los primeros teléfonos, estaban enfocadas en mejorar la productividad personal: se trataba de alarmas, calendarios, calculadoras y clientes de correo (Velazquez, 2014). 
Hubo un cambio grande con el ingreso de iPhone al mercado, ya que con él se generaron nuevos modelos de negocio que hicieron de las aplicaciones algo rentables, tanto para desarrolladores como para los mercados de aplicaciones, como App Store, Google Play y Windows Phone Store (Velazquez, 2014).

\section{Teclado QWERTY}

Atrás han quedado los tiempos en los que estábamos obligados a utilizar un teclado de dígitos en donde cada uno tenía 3 letras para escribir en nuestro celular. Aunque muchos celulares convencionales aún tienen esta característica los smartphones ya no. Los dispositivos inteligentes de hoy cuentan con teclado qwerty que es el formato que utilizan también los teclados de computadora y ofrecen una más cómoda experiencia al momento de estar escribiendo.

\section{Mensajería Multimedia}

Recuerdo cuando era algo de las películas ver como entre agentes policíacos se enviaban fotos de productos o escenarios en tiempo real y parecía ser la última maravilla posteriormente llegaron los mensajes multimedia pero realmente no resultaba atractivo. Hoy en día gracias a los smartphones podemos enviar a través de los servicios de mensajería imágenes de alta calidad y hasta videos en tiempo real.

Estas son las características más relevantes que definen hoy en día un Smartphone. La tecnología avanza tan rápido que posiblemente mañana sea otro y dentro de un mes otro. Es cierto, no sabemos qué es lo que sigue, pero eso sí, todos estos cambios y los beneficios que nos traen siempre serán bienvenidos.

Kit de Desarrollo de Software (SDK)

De acuerdo con 4rsoluciones (2013), un SDK (Software Development Kit), o kit de desarrollo de software, es un conjunto de herramientas que ayudan a la programación de aplicaciones para un entorno tecnológico particular. Es decir, las aplicaciones desarrolladas sobre el SDK estarán destinadas a algún sistema operativo, plataforma hardware, consola de videojuegos o paquete de software 
en especial. Son muchos los recursos que puede contener un SDK. Detallemos algunos de ellos:

- Una interfaz de programación de aplicaciones (API). Puede verse como una abstracción del funcionamiento interno del entorno sobre el que vamos a trabajar. Se trata de un conjunto de funciones, rutinas, estructuras de datos, clases y variables que nos permiten manipular el mecanismo de la plataforma sin conocerlo internamente.

- Un entorno de desarrollo integrado (IDE). Un editor que nos ayuda a escribir fácilmente el código fuente del programa. Generalmente, también brinda una interfaz amigable para dos aplicaciones fundamentales:

- Debugger. Permite testear el programa en cada paso de su ejecución.

- Compilador. Traduce el código fuente a lenguaje de máquina, obteniendo así un programa ejecutable.

- Código de ejemplo y otra documentación. Como punto de partida para empezar a desarrollar aplicaciones.

- Un emulador del entorno. Por ejemplo, si desarrollamos una aplicación para móviles desde una computadora de escritorio, nos permite saber cómo la vería el usuario final.
Actualmente, plataformas como los sistemas operativos Android, iOS y Windows Phone ofrecen kits para desarrollar software que funcione sobre sus entornos, y muchas redes sociales tienen SDK específicos para desarrollar todo tipo de aplicaciones en diferentes lenguajes (4rsoluciones, 2013)

\section{Sistema Operativo (SO)}

Tecnologia\&informática

(2018) Un sistema operativo puede ser definido como un conjunto de programas especialmente hechos para la ejecución de varias tareas, en las que sirve de intermediario entre el usuario y la computadora. Este conjunto de programas que manejan el hardware de una computadora u otro dispositivo electrónico. Provee de rutinas básicas para controlar los distintos dispositivos del equipo y permite administrar, escalar y realizar interacción de tareas.

Un sistema operativo, tiene también como función, administrar todos los periféricos de una computadora. Es el encargado de mantener la integridad del sistema. 
Podemos decir que el sistema operativo es el programa más importante de la computadora (Tecnologia \& informática, 2018).

Un sistema operativo (SO) tiene que lograr que el equipo (y los periféricos) puedan ser utilizados sin problemas por alguien que no conozca los detalles del hardware. Tiene que posibilitar que el usuario cree y acceda a los archivos, use los programas, ingrese a internet, juegue y todas las cosas que pueden ser hechas con las computadoras y/o dispositivos móviles. Además de eso, el sistema operativo tiene que ejecutar los programas y ayudarlos a acceder a los recursos del sistema de una forma simple y organizada (Tecnologia \& informática, 2018).

Existen muchos tipos de Sistemas Operativos, cuya complejidad varía dependiendo de qué tipo de funciones proveen, y en qué tipo de equipo puede ser usado. Algunos sistemas operativos permiten administrar gran cantidad de usuarios, en cambio otros controlan dispositivos de hardware como bombas de petróleo (Tecnologia \& informática, 2018).
Cuando el sistema operativo comienza a funcionar, inicia los procesos que luego va a necesitar para funcionar correctamente. Esos procesos pueden ser archivos que necesitan ser frecuentemente actualizados, o archivos que procesan datos útiles para el sistema. Es posible tener acceso a distintos procesos del sistema operativo, a través del administrador de tareas, donde se encuentran todos los procesos que están en funcionamiento desde la inicialización del sistema operativo hasta su uso actual (Tecnologia \& informática, 2018).

Una de las atribuciones del sistema operativo es cargar en la memoria y facilitar la ejecución de los programas que el usuario utiliza. Cuando un programa está en ejecución, el sistema operativo continúa trabajando. Por ejemplo, muchos programas necesitan realizar acceso al teclado, vídeo e impresora, así como accesos al disco para leer y grabar archivos. Todos esos accesos son realizados por el sistema operativo, que se encuentra todo el tiempo activo, prestando servicios a los programas que están siendo ejecutados (Tecnologia \& informática, 2018). 
El sistema operativo también hace una administración de los recursos de la computadora, para evitar que los programas entren en conflicto. Por ejemplo, el sistema operativo evita que dos programas accedan simultáneamente al mismo sector de la memoria, lo que podría causar grandes problemas. El sistema operativo funciona como un "maestro", procurando que todos los programas y todos los componentes de la computadora funcionen de forma armónica (Tecnologia \& informática, 2018).

\section{Aplicaciones (APPS)}

Cuello Javier y Vittone José (2017) nos dicen que, aunque no parezca, las aplicaciones llevan tiempo entre nosotros. Antes de empezar a diseñarlas, Las aplicaciones (también llamadas apps) están presentes en los teléfonos desde hace tiempo; de hecho, ya estaban incluidas en los sistemas operativos de Nokia o Blackberry años atrás. Los móviles de esa época contaban con pantallas reducidas y muchas veces no táctiles, y son los que ahora llamamos feature phones, en contraposición a los smartphones, más actuales.
En esencia, una aplicación no deja de ser un software. Para entender un poco mejor el concepto, podemos decir que las aplicaciones son para los móviles lo que los programas son para los ordenadores de escritorio. Actualmente encontramos aplicaciones de todo tipo, forma y color, pero en los primeros teléfonos, estaban enfocadas en mejorar la productividad personal: se trataba de alarmas, calendarios, calculadoras y clientes de correo (Velazquez, 2014).

Hubo un cambio grande con el ingreso de iPhone al mercado, ya que con él se generaron nuevos modelos de negocio que hicieron de las aplicaciones algo rentables, tanto para desarrolladores como para los mercados de aplicaciones, como App Store, Google Play y Windows Phone Store (Velazquez, 2014).

\section{Base de Datos para Aplicacio-} nes Móviles (SMBD)

Orozco Tapia Ana Maria (2012) Inicialmente las aplicaciones inalámbricas para dispositivos móviles eran programas totalmente desconectados de las empresas o siste- 
mas de computación. Esto desde el punto de vista en tiempo real. Estas aplicaciones eran por lo general Palm Pilots limitadas a libreta de direcciones, horarios, etc. Esta pequeña base de datos estaba bien para ese momento, pero a medida que avanza la tecnología, las personas necesitan más.

Surgen las redes inalámbricas, pero existe el problema de disponibilidad de dispositivos inalámbricos y teléfonos WAP (Wireles Application Protocol) que se encuentran limitados en memoria y el tamaño de la pantalla para mostrar la información, además que introducir información en estos dispositivos móviles puede llegar a ser bastante difícil por su tamaño. De todas formas, la tecnología es excelente, la habilidad que se tiene para conectarse al World Wide Web desde casi cualquier sitio en cualquier momento es una de las mayores ventajas que se tiene en la era de la información.

Con Internet inalámbrico, ya no es necesario estar físicamente frente al computador personas o dentro de las empresas para poderse co- nectar a las aplicaciones o las bases de datos. Con las portabilidades de la tecnología inalámbrica, nos podemos conectar a Internet o la intranet de la empresa para tomar datos almacenados. Estos datos están estructurados y organizados en entidades y objetos que se encuentran disponibles para los usuarios como información. La mayor ventaja se encuentra en que se le da la información al usuario en el mismo momento que es solicitada.

\section{Entornos de Programación para Desarrollar en Android \\ Basic 4 Android}

La plataforma Basic4Android es una de las grandes enemigas de Android Studio, que programa con el lenguaje VisualBasic, también rival de Java desde sus inicios. En este sentido, Basic4Android está especialmente indicado para aquello desarrolladores Android que empezamos a desarrollar apps en Android. La explicación la encontramos en que es un entorno más gráfico y menos abstracto, de manera que podemos ver los avances conforme programamos (Yeeply, 2015). 


\section{Mono para Android}

Aunque Java sea el lenguaje nativo de Android, la gran fama de este sistema operativo ha conseguido que llegaran herramientas para programar apps en Android conociendo otros lenguajes de programación de apps. Los lenguajes que Microsoft para desarrollar apps C\# y .NET han llegado también a Android. Si deseas utilizar este lenguaje seguramente ya utilizarás Visual Basic, por lo tanto, solo te queda descargar la SDK de Mono para Android y directamente podrás desarrollar apps en Android desde tu entorno Microsoft (Yeeply, 2015).

\section{App Inventor}

¿Y si no tienes un backgroud técnico? ¿NO sabes ni C\#, ni .NET, ni Java y ni oír hablar de Visual Basic? Pues entonces tenemos que recordarte App Inventor, una herramienta de la que te hemos hablado ya en nuestro blog. Esta es una plataforma desarrollada por Google Labs para que más gente se uniera a programar en Android. Es un entorno totalmente visual en el que no hace falta ni escribir una sola línea de código para desarrollar apps en
Android. Uno de los grandes inconvenientes es también su punto fuerte, la simplicidad y la rapidez a la hora de cómo crear una app para Android. Es completamente gratuito, solamente hay que descargar App Inventor en la web y empezar a crear apps (Yeeply, 2015).

\section{Live Code}

Imagina una plataforma en la que puedas desarrollar apps en Android, pero también en iOS, Linux, Windows, además de programar webs y para servidores desde una misma herramienta, desde LiveCode. Si ponemos como ejemplo que una aplicación que hemos decidido desarrollar en Android, pero que ahora queremos Ilevarla a Windows y a iOS para cubrir más mercado. Lo lógico es que pienses que debes contratar a un desarrollador para Windows y otro para iOS. Pero, aunque eso es lo más recomendable, lo cierto es que, si tienes cierto conocimiento de la herramienta LiveCode, además de programar en Android puedes hacerlo para esas otras plataformas (Yeeply, 2015). 


\section{MetodologíA}

Para la realización de este artículo se realizó una investigación a través de una revisión de fuentes bibliográficas tanto impresas como electrónicas sobre toda la información que se consideró necesario para comprender lo necesario sobre el tema que se desea profundizar, así como la información útil para comprender la posterior propuesta que se realizó.

Por ello la investigación se puede dividir en dos partes, la primera es la información que nos permite conocer más en profundidad acerca de los teléfonos inteligentes y su estructura y funcionalidades mientras que en la segunda parte se profundiza más en los temas relacionados al desarrollo de las aplicaciones móviles como lo pueden ser las herramientas necesarias para ello.

\section{Resultados}

La investigación, busca optimizar los canales de comunicación entre el alumno y el docente debido a que el método actualmente empleado presenta algunos problemas, se pretende solucionar esto a través de la creación de una aplicación para dispositivos móviles que se debe distribuir tanto al alumnado como a los docentes.

Para que la aplicación móvil pueda optimizar la comunicación la aplicación debe contener las siguientes funciones:

- Generar salas de chat con todos los integrantes de la experiencia educativa, así como su correspondiente docente.

- Contener un directorio con los datos esenciales de todo docente de la facultad.

- Poder mandar mensajes personales a cualquier docente de la facultad aun si este no imparte una experiencia educativa al alumno.

- Un perfil del estudiante con su información académica esencial.

- Un perfil de cada docente únicamente con información pertinente de la facultad para evitar cualquier información personal del mismo.

Con esta investigación, presentada en este artículo, se pretende aportar conocimiento del ámbito tecnológico referente al desarrollo móvil, actualmente considerada una industria en auge debido al amplio campo laboral docente. 
Dado que una mejor comunicación ayuda a brindar una mejora en la enseñanza dentro del plante, es por esto que busco convencer a las autoridades encargadas de implementar nuevos métodos para poder mejorar el nivel de educación obtenido al finalizar nuestra preparación profesional, sirviendo este proyecto para dar un enfoque más amplio sobre las ventajas que las tecnología existentes bien orientadas pueden dar grandes ventajas frente a otras instituciones educativas.

\section{CONCLUSIONES}

En la actualidad la tecnología se ha convertido en algo indispensable para la vida de todas las personas quienes a través de esta pueden facilitar la vida diaria permitiendo la realización de varias tareas de forma más rápida, eficiente e incluso de forma automática.

Una de las tecnologías que se encuentra más presente en la vida de todas las personas son los dispositivos móviles, dispositivos que como su nombre lo indica permiten a sus usuarios llevarlos a cualquier lugar para hacer uso de ellos y el más común y usado por las personas son los teléfonos inteligentes o Smartphones.

Hasta hace algunos años los teléfonos celulares nos permitían realizar las funciones más básicas e indispensables para lo que se consideraba un teléfono, pero debido al desarrollo de la tecnología permitió a los teléfonos móviles evolucionar y convertirse en algo completamente nuevo, permitiendo realizar muchas nuevas funciones útiles para las personas, debido a ello estos se han convertido en una necesidad para todas las personas.

Por ello es necesario conocer más a fondo sobre estas herramientas que usamos diariamente, conocer sobre los componentes y características de estos nos permite elegir de mejor forma nuestros propios equipos.

Como bien es conocido en la actualidad predominan dos plataformas en el mercado las cuales son Android y IOS, quienes se discuten constantemente el primer lugar de número de usuarios. 
En la inmensa cantidad de aplicaciones que podemos obtener de estas tiendas de aplicaciones podemos encontrar siempre alguna aplicación que satisfaga las necesidades de los usuarios, pero nunca nos tomamos la molestia de preguntarnos acerca del desarrollo de estas.

También necesitamos conocer más acerca de los entornos de desarrollo, los cuales son aplicaciones que le facilitan a los desarrolladores múltiples servicios y funciones que facilitaran la creación de las aplicaciones.

Como se ha mencionado en necesario remarcar la importancia de las tecnologías para solucionar los problemas que se tienen presentes en los planteles académicos, la implementación de nuevas tecnologías nos permitirá brindar un mejor ambiente para todos los integrantes, así como brindarnos soluciones a diversos de nuestros problemas.

De estos métodos se podían obtener problemas por parte de los alumnos así como por parte de los maestros, para mantener un contac- to, los alumnos normalmente tenían que tener inscrita una experiencia educativo con el profesor, de otra manera un alumno que desea comunicarse con algún maestro del cual nunca le haya impartido una experiencia se hace muy difícil su comunicación.

Otros problemas se relacionan entre los mismos estudiantes quienes no compartían la información con los demás en algunas ocasiones, otros casos algún estudiante se quedaba fuera de los grupos impidiéndole recibir dicha información. Con esta investigación se trata de recalcar la importancia del área de la programación.

\section{BIBLIOGRAFÍA}

4rsoluciones (2013). ¿Qué es un kit de desarrollo de software (SDK)?

Recuperado el 20 de junio de 2018 , de 4rsoluciones:

http://www.4rsoluciones.com/blog/qu e-es-un-kit-de-desarrollo-desoftware-sdk-2/

Alegsa (2018). Definición de aplicación móvil (app). Recuperado el día 16 de junio de 2018, de Alegsa:

http://www.alegsa.com.ar/Dic/aplicaci on_movil.php

Arimetrics (2018). Qué es el entorno de desarrollo. Recuperado el dia 18 de noviembre de 2018, de Arimetrics: https://www.arimetrics.com/glosariodigital/entorno-de-desarrollo 
Avison D.E. y Fitzgerald G. (2006), Information system development. Maidenhead: McGraw-Hill Education

Cuello Javier y Vittone José (2017) Diseñando apps para móviles. Recuperado el día 26 de julio de 2018, de appdesignbook: http://appdesignbook.com/es/conteni dos/las-aplicaciones/

Ecured (2018). Aplicación web.

Recuperado el día 12 de diciembre de 2018, de EcuRed:

https://www.ecured.cu/Aplicaci\%C3 \%B3n_web

Expansión (2018, 21 de febrero). En México el número de Mexicanos con acceso a internet subió a 71.3 millones. Recuperado el día 30 de junio de 2018, de Expansión: https://expansion.mx/tecnologia/2018 /02/20/en-mexico-hay-713-millonesde-mexicanos-con-acceso-a-internet

Gabriel (2018). Desarrollo de aplicaciones móviles. Recuperado el día 5 de junio de 2018, de okhosting: https://okhosting.com/blog/desarrollo -de-aplicaciones-moviles/

Gestion.pe (2016). Teléfonos inteligentes: Esta es la historia y la evolución de los smartphones. Recuperado el día 7 de junio de 2018, de gestión:

https://gestion.pe/tendencias/telefon os-inteligentes-historia-evolucionsmartphones-147278?foto $=6$

Morillo J. (S/Y). Entornos de programación móviles. Recuperado el día 7 de noviembre de 2018, de exabyteinformatica:

https://www.exabyteinformatica.com/ uoc/Informatica/Tecnologia_y_desarr ollo_en_dispositivos_moviles/Tecnol ogia_y_desarrollo_en_dispositivos_ moviles_(Modulo_3).pdf 